Review

\title{
Matrix Mechanics as Regulatory Factors and Therapeutic Targets in Hepatic Fibrosis
}

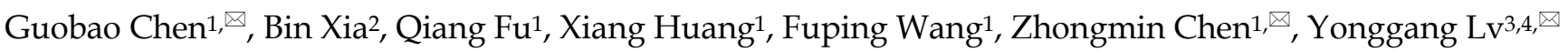 \\ 1. School of Pharmacy and Bioengineering, Chongqing University of Technology, Chongqing 400054, P. R. China. \\ 2. Chongqing Technology and Business University, Chongqing 400067, P. R. China \\ 3. Key Laboratory of Biorheological Science and Technology (Chongqing University), Ministry of Education, Bioengineering College, Chongqing University, \\ Chongqing 400044, P. R. China \\ 4. Mechanobiology and Regenerative Medicine Laboratory, Bioengineering College, Chongqing University, Chongqing 400044, P. R. China
}

$\square$ Corresponding authors: Dr. Guobao Chen, School of Pharmacy and Bioengineering, Chongqing University of Technology, No. 69 Hongguang Avenue, Banan District, Chongqing 400054, China. E-mail: gbchen@cqut.edu.cn. Dr. Zhongmin Chen, Professor, School of Pharmacy and Bioengineering, Chongqing University of Technology, No. 69 Hongguang Avenue, Banan District, Chongqing 400054, China. E-mail: chenzhongmin@cqut.edu.cn. Dr. Yonggang Lv, Professor, Mechanobiology and Regenerative Medicine Laboratory, Bioengineering College, Chongqing University, 174 Shazheng Street, Shapingba District, Chongqing 400044, China. E-mail: yglv@cqu.edu.cn.

() The author(s). This is an open access article distributed under the terms of the Creative Commons Attribution License (https://creativecommons.org/licenses/by/4.0/). See http:/ /ivyspring.com/terms for full terms and conditions.

Received: 2019.06.19; Accepted: 2019.08.07; Published: 2019.09.07

\begin{abstract}
The hallmark of liver fibrosis is excessive extracellular matrix (ECM) synthesis and deposition that improve liver matrix remodeling and stiffening. Increased matrix stiffness is not only a pathological consequence of liver fibrosis in traditional view, but also recognized as a key driver in pathological progression of hepatic fibrosis. Cells can perceive changes in the mechanical characteristics of hepatic matrix and respond by means of mechanical signal transduction pathways to regulate cell behavior. In this review, the authors first classify causes of liver matrix stiffening during fibrotic progression, such as higher degree of collagen cross-linking. The latest advances of the research on the matrix mechanics in regulating activation of HSCs or fibroblasts under two-dimensional (2D) and three-dimensional (3D) microenvironment is also classified and summarized. The mechanical signaling pathways involved in the process of hepatic matrix stiffening, such as YAP-TAZ signaling pathway, are further summarized. Finally, some potential therapeutic concepts and strategies based on matrix mechanics will be detailed. Collectively, these findings reinforce the importance of matrix mechanics in hepatic fibrosis, and underscore the value of clarifying its modulation in hopes of advancing the development of novel therapeutic targets and strategies for hepatic fibrosis.
\end{abstract}

Key words: liver fibrosis, matrix stiffness, targeting, mechanotransduction, myofibroblasts, hepatic stellate cells

\section{Introduction}

Liver fibrosis is a major cause of morbidity and mortality worldwide. Liver fibrosis occurs after a person experiences injury or inflammation in the liver. Many of the causes can lead to fibrosis, including autoimmune hepatitis, biliary obstruction, iron overload, nonalcoholic fatty liver disease, viral hepatitis B and C, and alcoholic liver disease [1]. Among them, liver fibrosis in Western societies is mainly caused by nonalcoholic fatty liver disease (NAFLD), while in China it is mainly caused by viral hepatitis. After liver fibrosis occurs, the cells, components, blood vessels, and microstructures in the liver will change in different degrees. Histologically, fibrosis is marked by disruption of the hepatic architecture and deposition of abnormal extracellular matrix (ECM). Among them, matrix stiffening is not only the pathological result of liver fibrosis, but also one of the important cues for promoting the progression of liver fibrosis. On the one hand, matrix stiffness, as an important feature and marker of pathological results of liver fibrosis, has received more and more attention and recognition in the clinical diagnosis of liver fibrosis. On the other hand, matrix mechanics has also been shown to promote the 
progression of liver fibrosis. Recently, studies have been proposed to target matrix mechanics as a new target for fibrosis treatment to explore new therapeutic approaches in liver fibrosis.

Myofibroblasts are the main effector cells that produce ECM in the process of fibrosis, and hepatic stellate cells (HSCs) are one of the main cell sources of myofibroblasts in the liver, accounting for about $82 \sim 96 \%$ [2]. The activation of HSCs is an important event in the process of hepatic fibrosis. After liver injury, quiescent HSCs are activated and differentiate into myofibroblasts. After excessive proliferation of myofibroblasts, a large amount of ECM such as collagen is synthesized, accompanied by increased matrix cross-linking and insufficient ECM degradation, eventually leading to liver fibrosis [3]. HSCs are non-parenchymal cells in the liver and are rich in lipid drops when they are in a quiescent state. As shown in Figure 1, once the liver is damaged, HSCs are activated and lose fat droplets, and their proliferative and contractile abilities increase and release cytokines such as inflammatory and fibrosis promotion [4]. HSCs will migrate after continuous activation, and a large amount of metalloproteinase tissue inhibitor-1 (TIMP-1) produced by autocrine and paracrine can effectively reduce the activity of Matrix metalloproteinase-2 (MMP-2) by binding to matrix MMP-2, thereby inhibiting the degradation of ECM by MMP-2, resulting in abnormal excessive deposition of ECM [5]. With the increasing pathological degree of hepatic fibrosis, the stiffness of hepatic tissue is increasing, the hepatic fibrosis in different pathological periods corresponds to different hepatic tissue stiffness [6, 7], and the stiffness of liver has become one of the important indexes in the clinical diagnosis of pathological staging of hepatic fibrosis.

The pathological stage of hepatic fibrosis is generally divided into 5 grades: F0 is non-hepatic fibrosis, F1 is mild hepatic fibrosis, F2 is moderate hepatic fibrosis, F3 is severe hepatic fibrosis, and F4 is cirrhosis. Corpechot et al. [8] examined the liver stiffness of 168 patients by transient elastography and found that the stiffness thresholds for different stages of liver fibrosis were $6.5 \pm 1.0 \mathrm{kPa}(\mathrm{F} 1), 8.1 \pm 0.7 \mathrm{kPa}(\mathrm{F} 2)$, $10.8 \pm 2.3 \mathrm{kPa}(\mathrm{F} 3)$, and $13.7 \pm 1.7 \mathrm{kPa}(\mathrm{F} 4)$, respectively. Georges and colleagues [9] examined liver stiffness at different stages of rat liver fibrosis and found that the shear modulus of the liver after fibrosis ranged from 3 $\mathrm{kPa}$ to $22 \mathrm{kPa}$, significantly higher than the shear modulus of normal liver. These findings all indicate that liver stiffness is significantly positively correlated with the degree of fibrosis.

Here we start with introducing various cues acting on liver matrix stiffening during the pathological progression, mainly including the changes of ECM, cell mechanics, and inflammation. We will describe the latest advances of the research on the matrix mechanics in regulating liver fibrosis under two-dimensional (2D) and three-dimensional (3D) microenvironment. Because accumulated evidences show that several signaling pathways are involved in the process of the liver matrix stiffening, such as the YAP/TAZ signaling pathway, which is

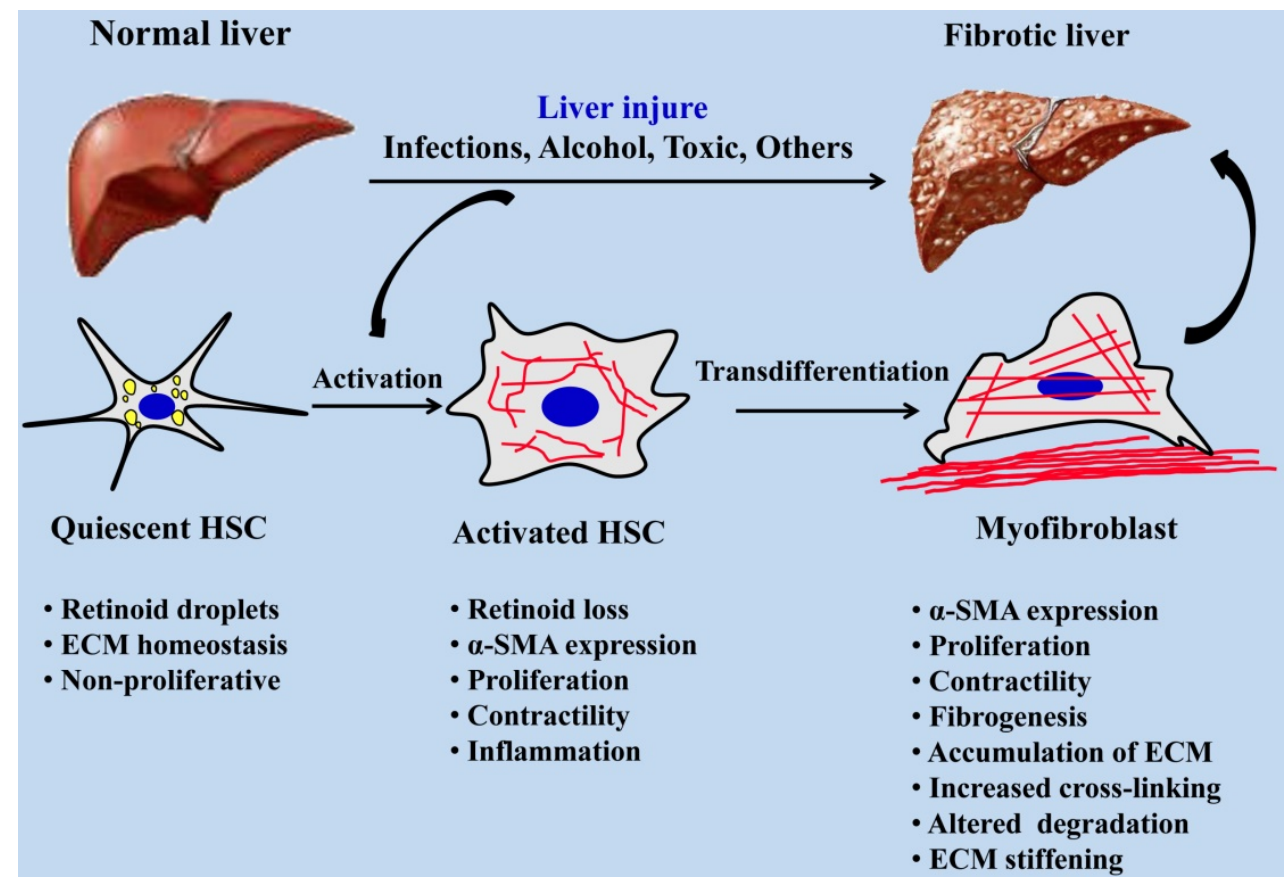

Figure 1. Activation process of quiescent HSCs into myofibroblasts during hepatic fibrosis. 
proved to play an important role during liver fibrosis. Finally, some potential therapeutic concepts and strategies based on matrix mechanics are described.

\section{Causes of Liver Matrix Stiffening during Fibrotic Progression}

There are many cues for the increase of hepatic matrix stiffness during the progression of fibrosis (Figure 2), including excessive deposition, organization and cross-linking of ECM, imbalance of MMPs and its inhibitors expression, increased stiffness of intrahepatic cells and hepatic inflammatory microenvironment. Stiffening of the liver matrix caused by these factors will further affect the fibrotic behavior of intrahepatic cells and form positive feedback.

\subsection{Excessive deposition of ECM, mostly collagen type I}

Many factors have been shown to lead to change in the stiffness of the liver. Among them, the major reason of elevated matrix stiffness during liver fibrosis is dysregulated matrix synthesis and remodeling. Normally, the most important structural ECM components in liver matrix are collagen, proteoglycans, laminin, fibronectin, and matricellular proteins [10], and the ECM is degraded and reformed in a balanced way to maintain tissue homeostasis. In advanced stage of hepatic fibrosis, the liver contains approximately 6 times more ECM than normal, including collagen type I, III, and IV, fibronectin, undulin, elastin, laminin, hyaluronan, and proteoglycans [11]. For instance, the low-density basement membrane-like matrix of the space of Disse

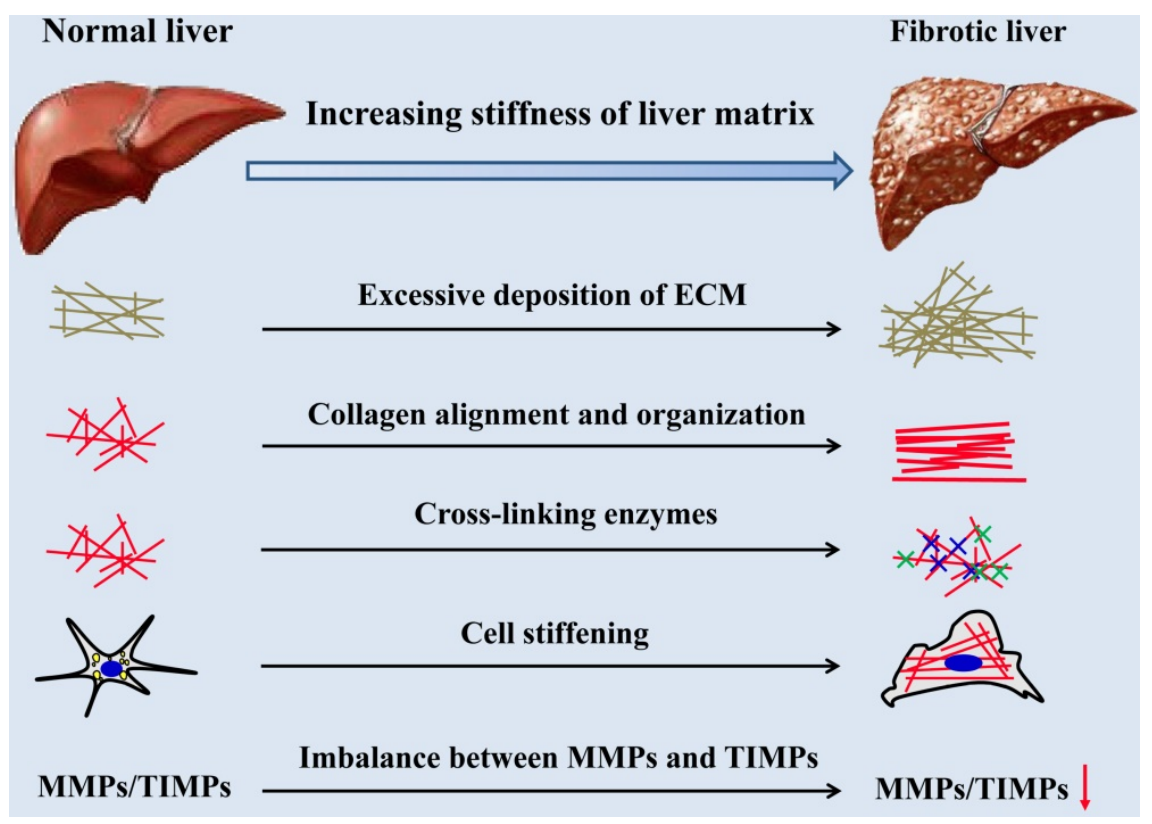

Figure 2. Causes of liver matrix stiffening during fibrotic progression. in normal liver is composed mainly of collagens IV and VI. After liver injury, disruption of this matrix and replacement by fibrillar collagens occur; this matrix is composed of collagen type I and III and fibronectin [1, 12]. In a carbon tetrachloride $\left(\mathrm{CCl}_{4}\right)$ induced rat liver fibrosis model, quantitative analysis of Sirius red staining revealed total collagen deposition in the liver increased as fibrosis progressed, which is $4.94 \%$ of the total tissue in liver sections at 4 weeks, $8.25 \%$ at 6 weeks, and $9.11 \%$ at 8 weeks [13]. Desai and colleagues [14] did the measurements orthogonal to fibrotic tracts and found that liver matrix was significantly stiffer in regions approaching fibrillar collagen deposition and returned to near normal rigidity in areas remote from it within the same lobule. Hepatic fibrosis is the results in excessive production of ECM, mostly collagen type I [15]. It has been proved that the content of collagen type I plays a key role in changing the mechanical characteristics of tissue and ECM, and the stiffness of various tissues and organs is positively correlated with the content of collagen type I $[16,17]$. Therefore, the massive accumulation of collagen type I is an important contributing factor in the local stiffening of the ECM in progressive liver fibrosis.

\subsection{Collagen alignment and organization}

In addition to change the amount of collagen deposited in the liver matrix, the alignment of the collagen fibrils is also contributing significantly to the alteration of the stiffness of liver matrix. Matrix stiffness can affect the contractility of growing cells and the cellular contractility further increase the contractility and alignment of the collagen fibrils in the liver matrix [18, 19]. Furthermore, the collagen matrix alignment in vivo can significantly improve the strength and stiffness of the entire matrix relative a matrix that is a disordered matrix [20]. The collagen matrix in the aligned region has greater stiffness due to its better structural order [21] and many in vitro studies prove that mechanical strength of ordered or aligned collagen nanofibers is significantly higher than that of the disordered nanofibers [22, 23]. For example, the electrospun collagen type I with the principal axis fibril alignment indicated an average load of $1.17 \pm 0.34 \mathrm{~N}$ at failure with a peak stress of $1.5 \pm 0.2 \mathrm{MPa}$. The average modulus for the 
longitudinal samples was $52.3 \pm 5.2 \mathrm{MPa}$. In cross fiber orientation, the peak load at failure was $0.75 \pm 0.04 \mathrm{~N}$ with a peak stress of $0.7 \pm 0.1 \mathrm{MPa}$. The modulus across the fiber long axis was $26.1 \pm 4.0 \mathrm{MPa}$ [23]. Another study also found significant differences in elastic modulus between the aligned and random collagen type I scaffolds, with the aligned groups (567 $\pm 134 \mathrm{kPa}$ ) having significantly greater elastic modulus than random groups $(349 \pm 85.5 \mathrm{kPa})$ [24]. Fibrosis is associated with alterations of interstitial flow because of vessel hyperpermeability and/or angiogenesis, $\mathrm{Ng}$ and colleagues [25] demonstrated that low levels of interstitial flow (0.1-0.3 dyn/ $\left.\mathrm{cm}^{2}\right)$ can induce collagen fibers to align perpendicular to the shear force direction and promote fibroblast transdifferentiation into matrix-producing myofibroblasts via triggering the expression of transforming growth factor- $\beta 1$ (TGF- $\beta 1$ ).

Besides the variation of orientation of collagen fibrils during the pathological progression of hepatic fibrosis, the change of collagen organization also found to be involved in the reversal of liver fibrosis, thus affecting liver matrix mechanics. Several researchers proposed that the large collagen fibers in liver matrix can break into smaller collagen fragments during the fibrosis regresses, and this change may further affect the local mechanical properties of liver matrix and make mechanical effects on the cells in liver matrix [26]. A new study, using atomic force microscopy (AFM) to compare the characteristics of normal and idiopathic pulmonary fibrosis lung tissue, found that the diameter of collagen fibers in the pulmonary matrix after fibrosis was significantly smaller, the swelling rate decreased, and the stiffness of the matrix increased significantly [27]. These findings suggest that the abnormal changes of collagen fibrils in liver matrix during fibrosis can remarkably regulate the mechanical properties of the tissue, and the underlying mechanism that lead to these abnormalities are crucial for researchers to understand of the fibrotic mechanisms and to provide new therapeutic targets.

\subsection{Collagen cross-linking enzymes and matrix mechanics}

In addition to the promotion of matrix stiffness by the increase in collagen content, the degree of cross-linking also affects the mechanical properties of the matrix. The collagen cross-linking enzymes appear to act a pivotal role in fibrosis or cancer progression by regulating the mechanical properties of the matrix [28]. In liver fibrosis, several studies have demonstrated that lysyl oxidase (LOX) may represent the major cross-linking activity for collagen $[9,29]$. LOX has also been shown to interact with fibronectin, which in turn increases the catalytic activity of LOX, thereby increasing the cross-linking of collagens, and subsequently, matrix stiffness [30]. The cross-linking endows the resistance to proteolytic degradation and further promotes excess deposition of ECM. Increased liver stiffness early after injury is associated with increases in LOX-mediated collagen cross-linking. Liu and colleagues [31] found that LOX had the functional contribution to stabilize collagen in liver fibrosis progression or reversal. In this work, the proportion of insoluble collagens increased from 5.7\% in healthy tissue to $14.7 \%$ and $19.1 \%$ in fibrotic tissue of $\mathrm{C} 57 \mathrm{Bl} / 6 \mathrm{~J}$ mice treated with $\mathrm{CCl}_{4}$ for 3 and 6 weeks, while the soluble collagens decreased from $92 \%$ in healthy controls to $84 \%$ and $79 \%$ in fibrotic tissue at 3 and 6 weeks. The treatment of LOX inhibitor $\beta$-aminopropionitrile (BAPN) decreases collagen stability during liver fibrosis progression and facilitates fibrosis reversal after $\mathrm{CCl}_{4}$-induced advanced liver fibrosis [31]. In another insightful work, BAPN-treated fibrotic mice approved the LOX inhibitor can effectively disrupt the collagen fibril networks and significantly reduce the stiffness of collagen fibril [32]. Besides the animal models, elevated expression and activity of LOX family members are also observed in sera of patients with hepatic diseases, for instance, liver fibrosis and cirrhosis [33].

Transglutaminases (TGases) are another type collagen cross-linking enzymes in progressive liver fibrosis [34, 35]. Some in vitro analyses verified that collagen type I gel cross-linked by the reactions of transglutaminase can effectively elevate the stiffness of gel compared to the uncrosslinked group [36, 37]. This treatment can not only improve the mechanical characteristics and stability of the tissue matrix, but also give the matrix resistance to proteolytic degradation. In an earlier study [34], the increase in transglutaminase detected by immunohistochemistry in fibrotic, compared to normal, liver is in agreement with the increase in transglutaminase expression during experimental liver fibrogenesis [38, 39]. Recently, Tatsukawa and colleagues [40] found that the TGase 1 activity may be involved in the functional modification of intracellular proteins, whereas TGase 2 activity contributes to the stabilization of extracellular proteins during liver fibrosis.

\subsection{MMPs and their specific inhibitors in liver matrix mechanics}

MMPs are a family of over 24 zinc-dependent endopeptidases capable of degrading virtually any component of the ECM, especially collagen that is responsible for matrix structure and mechanical properties in the ECM. The degradation has 
demonstrated that is a very critical process during the tissue or organ development, repair, fibrosis, and so on [41]. In normal livers, MMPs and their natural inhibitors, TIMPs, are always in a dynamic balance to maintain the normal expression and deposition levels of the ECM in the liver. In general, TIMPs are a family of at least four identified physiological inhibitors (TIMP-1, TIMP-2, TIMP-3, and TIMP-4) capable of regulating proteolytic activities of MMPs in tissues [42]. However, after fibrosis occurs, this balance will be broken and the profibrogenic effects of TIMP-1 are thought to be mediated via preventing collagen degradation through inhibition of MMPs [43]. The imbalance between the MMPs and TIMPs will affect the degradation and deposition of collagen, and these variations in ECM composition likewise have a profound influence on the mechanical characteristics of the liver matrix [44]. By detecting and comparing the liver stiffness and the expression of TIMPs in 29 patients with acute liver failure, the expression of hepatic stiffness and TIMP-1 in patients was found to be significantly higher than that of healthy controls [45]. Meanwhile, the compensatory increase in MMP expression is overcome by a parallel decrease in the MMP/TIMP ratio both during the onset and 7 days after the patients first presented with acute liver failure [45]. And in another study, Latronico and colleagues [46] measured the circulating levels of different MMPs and TIMPs in HCV, it was found that TIMP-1 levels were significantly higher in HCV subjects compared to healthy donors and were correlated with liver stiffness. The findings show that the expression of MMP and TIMPs will change in the process of hepatic fibrosis, thus affecting the content change of collagen and other ECM components in liver matrix, eventually altering the matrix stiffness of liver and affecting the process of hepatic fibrosis.

\subsection{Cell stiffening contributes to the matrix mechanical properties of liver}

Just as different tissues have their specific elastic modulus in the body, different cells also have unique stiffness in the tissue or organ. It has been found in previous studies that diseased cells are stiffer than healthy cells in certain pathologies [47-49]. For instance, the study found that the elevated aortic stiffness with aging is not only due to changes in the ECM, but also because of the increased stiffness of vascular smooth muscle cells in the old male monkeys [47]. Similarly, in a recent study on non-idiopathic pulmonary fibrosis, the fibroblasts derived from patients with this disease were found to have higher stiffness than their normal counterparts [48]. And in an in vitro model for liver cirrhosis, the stiffness of human hepatoma-derived HepG2 cells was significant increased after treatment with three known induction factors (collagen substrates, alcohol, and $\mathrm{CCl}_{4}$ ) [49]. Moreover, changes in the stiffness of hepatocytes can further affect the hepatic functions, such as glucose secretion [49]. In these studies, the increase in cell stiffness was mainly due to changes in the expression of the cytoskeletal proteins, for example, the increase in stiffness of vascular smooth muscle cells and lung fibroblasts was mainly due to the increased expression of a-smooth muscle actin (a-SMA). Another study also supports this conclusion from the side, that is, the normal lung can maintain $81 \%$ of the original stiffness of the liver after decellularization, while the lung with idiopathic pulmonary fibrosis retains only $44 \%$ of the original lung stiffness after decellularized treatment [50]. This finding shows that the cells in the fibrosis lungs contribute more to the stiffness of the whole lung than the cells in the normal lungs. During the fibrosis process, quiescent HSCs are activated to become myofibroblasts and expressed more a-SMA, so there is reason to believe that the stiffness of myofibroblasts may be higher than that of quiescent HSCs.

\subsection{Hepatic inflammation microenvironment and liver stiffness}

Although fibrosis is certainly a major contributing factor to liver stiffness, other characteristics of liver diseases, like inflammation may potentially affect liver stiffness. A recent study of pediatric patients with a variety of liver diseases similarly found that the correlation between alanine aminotransferase and transient elastography result was stronger among those with inflammatory diagnoses and F0/F1 fibrosis [51]. Inflammation is one of the most typical features of chronic liver disease of viral, alcoholic, fatty and autoimmune origin. It occurs at all stages of liver disease, including fibrosis, sclerosis and the development of liver cancer [52]. Previous studies have contributed to promote the understanding of the connection between liver inflammation and fibrosis [53, 54]. In a paper analyzing liver inflammation and liver stiffness by comparing 325 clinical samples, it was found that inflammation had a significant effect on liver stiffness values in patients with mild fibrosis, but not in patients with significant fibrosis [54]. Moreover, it was also found that patients with the same fibrosis stage but higher inflammation grade tended to have higher liver stiffness measurement values [54]. After the occurrence of hepatic fibrosis is often accompanied by intrahepatic inflammation, inflammation not only affects the function of the liver and immune microenvironment, inflammation caused by the secretion of intrahepatic ECM has also 
been shown to be involved in the changes in the stiffness of the intrahepatic matrix.

\section{Matrix Mechanics and Progression of Liver Fibrosis}

Given the important role that matrix mechanics plays in the progression and reversal of liver fibrosis, more and more researchers are beginning to focus on how to construct matrix microenvironment in vitro that can simulate occurrence and development of liver fibrosis in vivo, including 2D and 3D conditions. In the liver, the most studied cells in response to changes in matrix stiffness are HSCs and portal fibroblasts. These two types of cells are the major cellular sources of collagens and LOX in normal liver and early liver injury [55]. They can sensitively perceive and respond to alteration in the stiffness of the matrix in which they are located. Previous studies suggest that matrix stiffness affects multiple biological behaviors and properties of cells and tissues, including cell adhesion, proliferation, migration, differentiation, and so on [56, 57]. Therefore, analysis of the influences of matrix stiffness on the progression of liver fibrosis will provide the researchers with new insights in understanding pathological mechanisms and potential therapeutic targets for hepatic fibrosis.

\subsection{D matrix stiffness}

At present, most existing studies on engineering the matrix mechanical microenvironment of intrahepatic cells have been focused on 2D substrates. Polyacrylamide (PA) gel is the most classic and commonly used polymer to create elastic cell culture substrates with tunable stiffness by adjusting the acrylamide monomer and crosslinker content in PA gels $[58,59]$. The research team led by Wells has found that a stiffer matrix microenvironment can induce portal fibroblasts [60] and HSCs [61] to differentiate into myofibroblasts (Figure 3). Portal fibroblasts were cultured on PA gels support of variable stiffness (400 $\mathrm{Pa} 12 \mathrm{kPa}$ ) coated with thin layers of collagen type I to observe the myofibroblastic differentiation in different substrates [60]. It was found that the softest substrate $(400 \mathrm{~Pa})$ can maintain the phenotypic appearance of freshly isolated fibroblasts. Conversely, the substrate with a stiffness of $12 \mathrm{kPa}$ can promote the myofibroblastic differentiation of portal fibroblasts by elevating the expression of a-SMA at gene and protein levels. Moreover, the cell area also increases as the matrix stiffness increases. It was also found that TGF- $\beta$ and matrix stiffness play a synergistic role in promoting the mRNA expression of procollagens I and III [60]. Similar results were also found in HSCs cultured on PA gels of variable stiffness, ranging from 0.4 to $12 \mathrm{kPa}$ [61]. Activated
HSCs are thought to play a central role in secreting large amounts of ECM and regulating the degradation of matrix. The results showed that HSCs seeded on the softer substrates (0.4-1.0 $\mathrm{kPa}$ ) for one week could maintain their quiescent state, including rounded morphology and lipid droplets. On the stiffer substrates $(8-12 \mathrm{kPa})$, the lipid droplets of the HSCs disappeared, the morphology changed from circular to spreading, and a-SMA expression level and organization of stress fibers increased, indicating that the HSCs had activated into myofibroblasts [61]. Quiescent HSCs initially planted on the softer substrates were then covered with the glass coverslips for 3 days could increase cell spreading area and loss of lipid droplets. This finding indicated that HSCs on softer substrates were viable and the stiffness sensing was dynamic. The study also specifically pointed out that the substrate stiffness is more decisive than the chemical properties of substrate when determining HSCs phenotypes in vitro [61]. Exogenous TGF- $\beta$ addition is not sufficient to promote an increase in the level of required expression of HSCs on the softer substrates $(0.4 \mathrm{kPa})$. However, high-matrix stiffness was needed for the expression of a-SMA and collagen type I, no matter of TGF- $\beta$ exposure, although addition of TGF- $\beta$ significantly enhanced the effects of stiffness on the expression of a-SMA [61]. Therefore, the above findings under $2 \mathrm{D}$ conditions proved that change of liver matrix mechanics drives the pathological progression of fibrosis. Additionally, the studies also found that 2D matrix stiffness can effectively regulate the proliferation of hepatocellular carcinoma cells and chemotherapy tolerance. Increasing matrix stiffness $(12 \mathrm{kPa})$ promotes proliferation and resistances to chemotherapy, whereas a soft environment $(1 \mathrm{kPa})$ induces reversible cellular dormancy and stem cell characteristics in hepatocellular carcinoma [62].

In addition to the classic PA hydrogels for the preparation of different stiffness substrates, the research team led by Wells and Burdick using methacrylates modified hyaluronic acid macromolecules and sequence of addition of dithiothreitol and then crosslinking by UV light induced radical polymerization to construct different stiffness substrates to mimic normal and fibrotic livers and investigate the effects and mechanism of soft or stiff substrates on the activation of HSCs [63-65]. It was found that the high stiffness hydrogel substrate $(24 \mathrm{kPa})$ can significantly increase the spreading area of rat HSCs, loss of lipid droplets, expression of high levels of a-SMA and collagen type I, and the low stiffness hydrogel substrate $(2 \mathrm{kPa})$ can maintain lipid droplets of HSCs and expresses high levels of peroxisome proliferator-activated receptors $\mathrm{Y}$ 
(PPARY) [63]. Additionally, considering that the mechanical microenvironment of the cells in the fibrosis process is dynamic, this research group also innovatively studied the effects and mechanisms of dynamic matrix stiffening [64] or softening [65] on the progression and reversal of liver fibrosis. The stiffening from $1.75 \mathrm{kPa}$ to $33.0 \mathrm{kPa}$ of matrix resulted in myofibroblast activation of HSCs, which is a common feature of pathological fibrosis. They also revealed that the timing of substrate stiffening strongly influenced cell fate and delayed stiffening further promoted myofibroblast activation [64]. Similarly, when the liver matrix becomes soft, it may also play an important role in the reversal of liver fibrosis. HSCs were cultured on tissue culture plastic obtained a myofibroblast phenotype and then maintained the activated phenotype onto the stiff hydrogel substrate $(20 \mathrm{kPa})$ [65]. Compared to the cells on the stiff control $(20 \mathrm{kPa})$, when the activated cells are cultured on stiff-to-soft ( $20 \mathrm{kPa}$ to $3 \mathrm{kPa}$ ) hydrogel substrate, the area of the cells is reduced and the expression levels of a-SMA and Yes-associated protein/Transcriptional coactivator with PDZ-binding motif (YAP/TAZ) are also decreased at 2 weeks. This result indicates that when the environment in which the activated cells grow from stiff to soft, the activated cells are reversed to a certain extent, but this reversal is not complete. Together, these findings demonstrated that matrix mechanics have great effects on fibrosis regression and reversal and that simulating the dynamically changing mechanical signals can help to better mimic the real environment of cells in vivo.

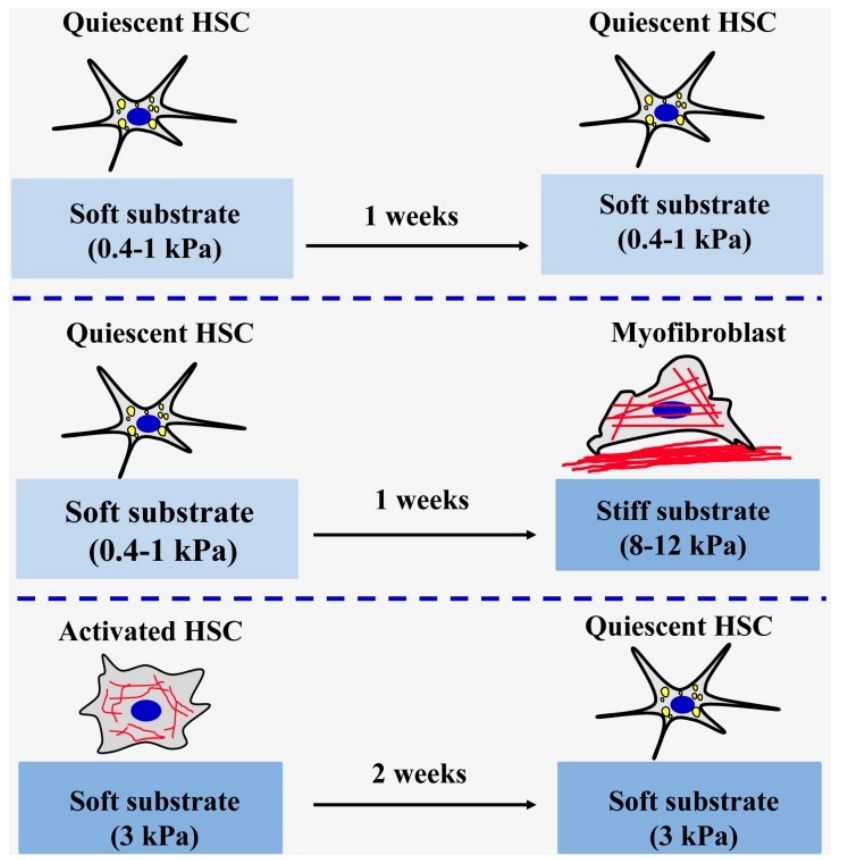

Figure 3. The effect of matrix stiffness on the activation of HSCs.
Besides the matrix stiffness sensing by HSCs in pathologic liver matrix stiffening, HSCs can also sense and response the matrix proteins in their culture system. Saums et al. [66] showed a new cell culture system combining layer-by-layer (LbL)-assembled films of different native matrix proteins with mechanically tunable substrates and to investigate the role of a role for both the matrix environment and substrate stiffness in myofibroblastic differentiation of HSC.

\subsection{D matrix stiffness}

Cells are exposed to a solid microenvironment that fully surrounds them in 3D environments, while cells in 2D platform are typically exposed to a solid, flat surface on the basal side and to liquid at the apical surface. To recapitulate intrahepatic cells behaviors and responses in both physiological and pathological conditions of liver, engineering 3D spatiotemporal matrix mechanical microenvironments in vitro may help investigators to explore and discover the relationship between the matrix mechanical characteristics of the liver and its fibrosis occurrence and reversal.

To investigate the relationship between 3D matrix stiffness and proliferation, survival of hepatic cells, Bomo and colleagues [67] fabricated the 3D collagen matrices with different stiffness to explore the responses of human hepatocarcinoma cells (Huh7) to them. Collagen type I scaffolds with two different stiffness were formed by controlling the concentrations of collagen solution. It was found that the concentrations of $0.75 \mathrm{mg} / \mathrm{mL}$ and $1.5 \mathrm{mg} / \mathrm{mL}$ were used to form the stiffness of $1 \mathrm{kPa}$ (soft scaffold) and $3.5 \mathrm{kPa}$ (stiff scaffold), respectively. The increase from $1 \mathrm{kPa}$ to $3.5 \mathrm{kPa}$ can be a good simulation of the elevation of liver matrix stiffness during the liver fibrosis development in vivo [67]. After 3 days of culture, Huh7 cells on the 3D stiff matrix were non-spread and exhibited spherical clusters. On the contrary, cells appeared a good spreading growth and developed monolayer-like structures on the soft collage matrix. Cells' spreading under 3D matrix stiffness is exactly opposite to the stiffness of the 2D matrix, which is directly related to the different ways in which cells are in contact with the matrix in under these two dimensions. Moreover, the survival and proliferation of Huh7 cells on different stiffness in 3D collage matrix were also evaluated in this work. It is found that the proliferation of Huh7 cells and normal hepatocytes on stiff collage matrix is higher than that in the soft collage matrix and 2D control by the colorimetric MTT assay. Furthermore, the stiff matrix can increase the biotransformation activities of Huh7 and Human hepatocytes in $3 \mathrm{D}$ as compared to $2 \mathrm{D}$ 
cultures and 3D soft matrix [67]. This study points out the different effects of matrix stiffness on liver cells under 2D and 3D microenvironments, but the difference between the collagen scaffold and the composition of the liver matrix under physiological or pathological conditions is still large, and the 3D scaffold systems which can better simulate the microenvironment of hepatic matrix mechanical microenvironments need to be further developed. Ma and colleagues [68] used the liver decellularized ECM and a rapid digital light processing (DLP)-based 3D printing method to construct a new 3D liver matrix with tailored mechanical features to mimic the pathological mechanical properties of hepatocellular carcinoma. Mechanical characteristics of these 3D scaffolds can be modulated by controlling the light exposure time. Particularly, three different exposure times of $10 \mathrm{~s}, 20 \mathrm{~s}$, and $40 \mathrm{~s}$ were selected to fabricate 3D scaffolds with stiffness values of $0.5 \mathrm{kPa}, 5 \mathrm{kPa}$, and $15 \mathrm{kPa}$, which each corresponds to the softer than healthy liver range (soft), healthy liver range (medium), and cirrhotic range (stiff), respectively. The influence of scaffold composition can be eliminated by adjusting the light exposure time to change the stiffness of the scaffold. Compared with low stiffness and medium stiffness, the study found that HepG2 cells were encapsulated in high-stiffness scaffolds for 3 and 7 days to limit cell viability and growth. In addition, the stiffness of the scaffold has also been found to regulate cell migration and invasion by regulating the expression of cellular MMP2 and MMP9, that is, HepG2 in the high-stiffness scaffold endocrine more MMP2 and MMP9, thus further promoting the potential migration and invasion ability of HepG2 [68]. This nature-derived ECM scaffolds with regionally matrix mechanical features can provide the natural composition, biomimetic structure and more physiologically relevant tissue mechanical properties to serve as a better platform for liver disease research. In addition to directly acting on intrahepatic cells to affect liver fibrosis, matrix stiffness has also been found to promote HSCs activation by regulating angiogenesis, thus affecting the process of hepatic fibrosis. Recently, a research group led by $\mathrm{Du}$ [69] has constructed a 3D microfibrotic niche to mimic fibrotic liver matrix and utilized them to investigate how matrix mechanics affect the formation of capillary-like structures and its influence on the progression of liver fibrosis. This 3D microfibrotic niche consisted of liver sinusoid endothelial cells (LSECs), cultured on an underlying 2D polyethylene glycol (PEG) hydrogel substrate with varying stiffness, and an overlaid 3D collagen type I hydrogel, which was embedded with HSCs. It was found that the softer PEG hydrogel substrates with stiffness in the range 140-610 Pa showed typical pro-angiogenic properties as seen in early-stage fibrosis, whereas the stiffer ones $(>1.2 \mathrm{kPa})$ exhibited random migration, sparsely distributed focal adhesion complexes and leaky vessels as observed during late-stage fibrosis [69, 70]. Importantly, the stiffness of early-stage 3D microfibrotic niche was significantly increased by condensing of collagen fibrils by angiogenesis, and resulted in activation of HSCs by LSECs. Liu and colleagues [69] found the activation of HSCs by the mechanical force via interactions with collage fibrils and a cell membrane-bound discoidin domain receptor 2 (DDR2) receptor by using in vitro and in vivo models. The innovative fibrotic microniches developed and the findings from this excellent research work highlight the dynamic properties of fibrosis progression and the contribution of angiogenesis to fibrosis in the environment of different matrix mechanical signals.

\section{Matrix Mechanics and Mechanotransduction Pathways in Liver Fibrosis}

In this pathological process of gradual stiffening of the hepatic matrix, the cells in the liver matrix perceive changes in the biophysical characteristics of their microenvironment and activate the mechanotransduction pathways, thus converting the external mechanical stimuli into biochemical signals, ultimately guiding cells behaviors. How matrix mechanics influences and regulates cell fate from the macroscopic to the microscopic scales is still a black box that is not fully open, but some studies have found that some proteins involved in the mechanotransduction pathways play a role in this process.

As a classical signaling pathway, the TGF-Smad signaling pathway has been shown to be an important signaling pathway involved in the progression of fibrosis in matrix stiffness regulation. Myofibroblasts contraction can release the latent TGF- $\beta 1$ from latent stores in local stiff matrix and still remains latent in the comparably soft matrix. Because the deformation caused by cell contraction on a soft substrate is absorbed and protected the large potential complex against conformational changes, and on a stiff substrate, this deformation is transmitted by integrin to induce the release of TGF- $\beta 1$ after deformation of the large potential complex [71, 72]. The released TGF- $\beta 1$ feeds back by binding to its receptor and promotes development of fibrosis. Because activated TGF- $\beta 1$ is a potent fibrogenic cytokine that promotes myofibroblastic differentiation, this finding suggests that stiff matrix may regulate myofibroblastic 
differentiation through an extrinsic mechanotransduction pathway in which stress fiber-generated contractile forces in response to matrix stiffening are transduced across the cell membrane and converted into the fibrogenic signal by activation of latent TGF- $\beta 1$, resulting in myofibroblastic differentiation [73].

The activity of Rho-associated protein kinase (ROCK) of fibrosis fibroblasts has been shown to be increased by matrix stiffening [74, 75], and the contraction of myofibroblasts is dependent on Rho/ROCK/myosin light chain pathway activation [76] RhoA expression of lung fibroblasts at the mRNA level and protein level was higher on the stiff matrix $(20.80 \pm 2.52 \mathrm{kPa})$ by the elevated stiff matrix than that in the soft matrix $(0.52 \pm 0.09 \mathrm{kPa})$ [74]. Consistent with results of RhoA, a stiff matrix also promotes the expression of a-SMA at gene and protein level and induces myofibroblast differentiation. Recently, Dou et al. [77] also demonstrated that substrate stiffness can activated AKT signal pathway by RhoA to induce phosphorylation of p300 and translocate to the nucleus of HSCs and affect the tumor metastasis by increasing expression of paracrine factors such as CXCL12. In this process, the protein expression of RhoA of human HSCs was increased by the matrix stiffness from $0.4 \mathrm{kPa}$ to $25.6 \mathrm{kPa}$ and promoted the nuclear accumulation of p300 and HSCs activation, suggested that RhoA is required of stiffness-induced phosphorylation of p300 [77].

Recently, the YAP-TAZ signaling pathway is proved to be another important signaling pathway to participate in the progress of matrix stiffness regulation of hepatic fibrosis [64, 78-80]. In liver fibrosis, YAP is activated in HSCs in response to matrix stiffening [64-65, 80]. In mouse fibrotic livers, the stiffened matrix causes the integrin clustering and then activates the kinase activity of focal adhesion kinase (FAK). The activated FAK signal stimulates the ROCK-dependent actin remodeling and the formation of stress fibers. Finally, ROCK-myosin-II mediates the contraction of activated HSCs or fibroblasts and then drives the translocation of YAP-TAZ from the cytoplasm into the nucleus [81]. The activation of YAP-TAZ in the nucleus results in inducing the expression of profibrotic genes and increasing the expression of a-SMA and excessive matrix deposition, which are associated with the generation of myofibroblasts [78]. Immunohistochemical examination of fibrotic areas of livers from patients with hepatitis $C$ [80] and lung tissue from patients with idiopathic pulmonary fibrosis (IPF) [82] found that the myofibroblasts in the fibrotic region with strong nuclear staining of YAP and TAZ. By analyzing the local stiffness of continuous slices of fibrosis tissue and the immunostaining of TAZ, the researchers also found that the part of TAZ positive nucleus and the areas of elevated tissue stiffness were co-localized. The findings demonstrated that increased stiffness of fibrotic tissue in vivo can promote YAP-TAZ nuclear accumulation and transcription activity in activated HSCs or myofibroblasts, promote collagen and fibronectin deposition and cause ECM stiffening, and eventually form a positive feedback loop with negative consequences [82].

\section{Targeting the Matrix Mechanics in Liver Fibrosis}

Matrix stiffening is a defining characteristic of hepatic fibrosis. While traditionally viewed as an endpoint, matrix stiffening is now recognized to develop early during fibrosis initiation, and to contribute prominently to disease progression through mechano-activation of myofibroblasts derived from fibroblasts or HSCs. Based on the importance of matrix mechanics in the current process of liver fibrosis, studies have been conducted to investigate whether targeting liver matrix mechanics has the potential to treat or even reverse liver fibrosis.

\subsection{Reduce or reverse the stiffness of the liver matrix}

Obviously, the most direct way to target the stiffness of the hepatic matrix is to inhibit and slow the increase of liver stiffness in the process of hepatic fibrosis or even reduce the stiffness of liver until it returns to normal liver stiffness. From this point of view, by influencing or reducing the factors that can promote the stiffening of the liver matrix, the purpose of reducing the stiffness of the liver matrix can be achieved, thereby inhibiting cell activation, alleviating or reversing liver fibrosis. Firstly, the purpose of reducing the stiffness of the liver is achieved by targeting the degree of cross-linking of the liver matrix. The LOX family of enzymes plays important roles in collagen cross-linking, contributing to both the increase in tissue stiffness and degradation resistance of collagen-rich matrices. Previous preclinical models reveal a striking reduction in tissue stiffness and fibrosis with use of the nonspecific LOX inhibitor BAPN [83]. In addition to direct targeted LOX studies, inhibition of lysyl oxidase-like-2 (LOXL2) raised during hepatic fibrosis has also been shown to be effective in relieving hepatic fibrosis and promoting fibrosis reversal [84, 85]. LOXL2 was shown to mediate the crosslinking of collagen and the fibrotic matrix stabilisation during the hepatic fibrosis process [85]. The degree of cross-linking and stability of collagen directly modulates the stiffness of the 
entire liver matrix, thereby affecting the activation or inactivation of hepatic progenitor cell (HPC). It was direct assessed using a stepwise collagen extraction assay and found that the anti-LOXL2 antibody AB0023 treatment significantly suppressed the increase in crosslinked collagen, with a $45 \%$ reduction in insoluble fraction between 6 and 12 weeks of thioacetamide (TAA) administration and a 20.8\% reduction of overall crosslinked collagen, compared with the control [85]. LOXL1, another member of the LOX family, is thought to have the function of promoting the crosslinking of elastic proteins [86]. Recently, a new study has confirmed that suppressing the expression of LOXL1 through silencing techniques can prevent the progression of cirrhosis by reducing the crosslinking of elastin in the hepatic matrix [87].

In addition, collagen, as one of the main components in the fibrotic matrix, plays a pivotal role in regulating the mechanical characteristics of the hepatic matrix. Therefore, targeting to reduce the synthesis of collagen [88] in the liver matrix during fibrosis or to degrade the excessive deposition of collagen by collagenase [89] has the potential to reduce the stiffness of hepatic matrix and achieve the goal of slowing down the process of hepatic fibrosis.

\subsection{Reduce or inhibit the response of cells to stiffening of the liver matrix}

Cells can sense and respond to the stiffness of their surrounding matrix [90]. In liver, HSCs or fibroblasts can be activated by the elevated stiffness of matrix, in turn, upregulates ECM deposition and stiffness, constituting a positive-feedback loop [91]. Therefore, it may be a potential way to ameliorate fibrosis by interfering with, reducing or inhibiting the responses of mechanosensitive cells in the liver to the stiffening of the matrix [92]. The small Rho GTPases provide a functional linkage between mechanosensing through integrin-based adhesion and the actomyosin cytoskeleton by generating traction forces. ROCK is the major downstream effector of Rho that drives cell contractility and is a mediator of fibrotic pathologies. The ROCK activity of fibroblasts can be activated by increasing the stiffness of lung matrix during fibrosis, inducing the differentiation of fibroblasts into myofibroblasts, increasing the expression of a-SMA, and enhancing the actin cytoskeletal polymerization [74, 93]. ROCK-mediated effects during fibrosis are mechanosensitive. Therefore, the inhibition of ROCK by means of drugs or inhibitors can alleviate the fibrosis process caused by the elevated matrix stiffness, which disrupts or blocks the responses of cells to tissue stiffening. Several studies have confirmed the inhibition of ROCK signaling by its inhibitors (such as Y-27632 and fasudil) attenuates stiffness-induced a-SMA expression and fiber assembly in myofibroblasts [74, 93-94].

After the initiation of fibrosis, YAP/TAZ will be activated by matrix stiffness and further promote the transformation of fibroblasts or HSCs into myofibroblasts, increase the deposition of collagen by the positive feedback loop that elevates the stiffness of matrix microenvironment and leads to YAP activation [80]. Knockdown of YAP levels by transfecting siRNAs or pharmacological inhibition of YAP by verteporfin can effectively inhibit the activation of HSCs in vitro and can prevent the fibrogenesis in a $\mathrm{CCl}_{4}$-induced liver fibrosis in mice [80]. Omega-3 polyunsaturated fatty acids ( $\omega-3$ PUFAs), such as docosahexaenoic acid (DHA) and eicosapentaenoic acid (EPA), demonstrate promising efficacy in alleviating hepatic fibrosis by targeting YAP to reduce the expression of pro-fibrogenic genes in activated HSCs and fibrotic liver [95]. The findings demonstrated that YAP activation undertakes a central role of YAP/TAZ in the fibrogenic cascade, and the selection of a suitable method to target nuclear transcription of YAP/TAZ in cells during fibrosis may also be a potential anti-fibrotic pathway.

Enhanced contractility of myofibroblasts is an important phenotypic feature of myofibroblast differentiation during fibrosis. Meanwhile, quiescent tissue fibroblasts become activated to a contractile, myofibroblast matrix secreting phenotype and ultimately increase the tissue stiffness [96]. For instance, Torr and colleagues [97] found that collagen matrix formation by myofibroblasts is dependent on the assembly of a fibronectin matrix and the assembly of fibronectin fibril by myofibroblasts requires contractile genes expression. Contractile genes expression is a necessary condition for the development of a contractile phenotype in myofibroblasts. Among them, the a-SMA gene is a key contraction gene that is raised in myofibroblasts and the expression of a-SMA may be the key to promote the accelerated assembly of fibronectin matrix by myofibroblasts [97]. Therefore, disruption of the a-SMA by siRNA knockdown affects the contraction of myofibroblasts, thus reducing the assembly of fibronectin matrix into fibrillary ECM. In addition, a recent study in airway fibrosis has also found that by simultaneously utilizing relaxin and LOX inhibitors to target myofibroblastic contractility and matrix stiffness, respectively, showed that the deposition of collagen could be significantly reduced and the re-epithelialization of remodeled airways could be promoted [98]. 


\section{Conclusion and Future Outlook}

Although previous studies confirm that several mechanically sensitive proteins are involved in the progression of liver fibrosis, these key proteins are commonly expressed in many tissues and organs of the body, participating in other normal physiological activities of the human body, inhibiting or blocking them may cause other diseases or dysfunction in the body. Therefore, the development of higher specificity and more accurate targeted drugs or small molecular inhibitors is still a key and difficult point in this field. As an important parameter for the progress of liver fibrosis, non-invasive and accurate detection of local stiffness of the liver matrix in vitro is one of the important prerequisites for the development of diagnosis and treatment targeting liver matrix mechanics, especially in the initial stage when the hepatic matrix begins to stiffen. Direct intervention or reduction of the stiffness of the liver matrix by appropriate physical or chemical methods, reducing or blocking the promotion of liver fibrosis by matrix mechanics may avoid the off-target effect of the use of drugs to some extent. Considering the heterogeneity of liver matrix mechanics, future studies also need to construct a more accurate culture platform in vitro that matches the mechanical heterogeneity of the liver matrix to facilitate the study of liver fibrosis and provide more accurate pharmaceutical screening platform.

\section{Acknowledgements}

This work was supported by grants from the National Natural Science Foundation of China (11702044, 11872134, 11672051, and 11902061), and Scientific Research Foundation of Chongqing University of Technology (2019ZD49).

\section{Competing Interests}

The authors have declared that no competing interest exists.

\section{References}

1. Hernandez-Gea V, Friedman SL. Pathogenesis of liver fibrosis. Annu Rev Pathol. 2011;6:425-56.

2. Mederacke I, Hsu CC, Troeger JS, Huebener P, Mu X, Dapito DH, et al. Fate tracing reveals hepatic stellate cells as dominant contributors to liver fibrosis independent of its aetiology. Nat Commun. 2013;4:2823.

3. Krizhanovsky V, Yon M, Dickins RA, Hearn S, Simon J, Miething C, et al. Senescence of activated stellate cells limits liver fibrosis. Cell. 2008;134:657-67.

4. Friedman SL. Evolving challenges in hepatic fibrosis. Nat Rev Gastroenterol Hepatol. 2010;7:425-36.

5. Ramezani-Moghadam M, Wang J, Ho V, Iseli TJ, Alzahrani B, Xu A, et al. Adiponectin reduces hepatic stellate cell migration by promoting tissue inhibitor of metalloproteinase-1 (TIMP-1) secretion. J Biol Chem. 2015;290:5533-42.

6. Ziol M, Handra-Luca A, Kettaneh A, Christidis C, Mal F, Kazemi F, et al. Noninvasive assessment of liver fibrosis by measurement of stiffness in patients with chronic hepatitis C. Hepatology. 2005;41:48-54

7. Yin M, Talwalkar JA, Glaser KJ, Manduca A, Grimm RC, Rossman PJ, et al. Assessment of hepatic fibrosis with magnetic resonance elastography. Clin Gastroenterol Hepatol. 2007;5:1207-13.
8. Corpechot C, Gaouar F, El Naggar A, Kemgang A, Wendum D, Poupon R, et al. Baseline values and changes in liver stiffness measured by transient elastography are associated with severity of fibrosis and outcomes of patients with primary sclerosing cholangitis. Gastroenterology. 2014;146:970-9.

9. Georges PC, Hui JJ, Gombos Z, McCormick ME, Wang AY, Uemura M, et al. Increased stiffness of the rat liver precedes matrix deposition: implications for fibrosis. Am J Physiol Gastrointest Liver Physiol. 2007;293:G1147-54.

10. Uygun BE, Soto-Gutierrez A, Yagi H, Izamis ML, Guzzardi MA, Shulman C, et al. Organ reengineering through development of a transplantable recellularized liver graft using decellularized liver matrix. Nat Med. 2010;16:814-20.

11. Bataller R, Brenner DA. Liver fibrosis. J Clin Invest. 2005;115:209-18.

12. Zhang Y, Ghazwani M, Li J, Sun M, Stolz DB, He F, et al. MiR-29b inhibits collagen maturation in hepatic stellate cells through down-regulating the expression of HSP47 and lysyl oxidase. Biochem Biophys Res Commun. 2014;446:940-4.

13. Vassiliadis E, Larsen DV, Clausen RE, Veidal SS, Barascuk N, Larsen L, et al. Measurement of CO3-610, a potential liver biomarker derived from matrix metalloproteinase-9 degradation of collagen type iii, in a rat model of reversible carbon-tetrachloride-induced fibrosis. Biomark Insights. 2011;6:49-58.

14. Desai SS, Tung JC, Zhou VX, Grenert JP, Malato Y, Rezvani M, et al. Physiological ranges of matrix rigidity modulate primary mouse hepatocyte function in part through hepatocyte nuclear factor 4 alpha. Hepatology. 2016 Jul;64(1):261-75.

15. Kisseleva $\mathrm{T}$, Brenner DA. Inactivation of myofibroblasts during regression of liver fibrosis. Cell Cycle. 2013;12(3):381-2.

16. Swift J, Ivanovska IL, Buxboim A, Harada T, Dingal PC, Pinter J, et al. Nuclear lamin-A scales with tissue stiffness and enhances matrix-directed differentiation. Science. 2013;341(6149):1240104.

17. Swift J, Discher DE. The nuclear lamina is mechano-responsive to ECM elasticity in mature tissue. J Cell Sci. 2014;127(Pt 14):3005-15.

18. Chiquet M, Renedo AS, Huber F, Flück M. How do fibroblasts translate mechanical signals into changes in extracellular matrix production? Matrix Biol. 2003;22(1):73-80.

19. Rens EG, Merks RMH. Cell contractility facilitates alignment of cells and tissues to static uniaxial stretch. Biophys J. 2017;112(4):755-66.

20. Lynch HA, Johannessen $\mathrm{W}$, Wu JP, Jawa A, Elliott DM. Effect of fiber orientation and strain rate on the nonlinear uniaxial tensile material properties of tendon. J Biomech Eng. 2003;125(5):726-31.

21. Provenzano PP, Inman DR, Eliceiri KW, Trier SM, Keely PJ. Contact guidance mediated three-dimensional cell migration is regulated by Rho/ROCK-dependent matrix reorganization. Biophys J. 2008;95(11):5374-84.

22. Islam A, Mbimba T, Younesi M, Akkus O. Effects of substrate stiffness on the tenoinduction of human mesenchymal stem cells. Acta Biomater. 2017;58:244-53.

23. Matthews JA, Wnek GE, Simpson DG, Bowlin GL. Electrospinning of collagen nanofibers. Biomacromolecules. 2002;3(2):232-8.

24. Lowe CJ, Reucroft IM, Grota MC, Shreiber DI. Production of highly aligned collagen scaffolds by freeze-drying of self-assembled, fibrillar collagen gels. ACS Biomater Sci Eng. 2016;2(4):643-51.

25. $\mathrm{Ng} \mathrm{CP}, \mathrm{Hinz} \mathrm{B}$, Swartz MA. Interstitial fluid flow induces myofibroblast differentiation and collagen alignment in vitro. J Cell Sci. 2005;118(Pt 20):4731-9.

26. Wells RG. Location, location, location: Cell-level mechanics in liver fibrosis. Hepatology. 2016;64(1):32-3.

27. Jones MG, Andriotis OG, Roberts JJ, Lunn K, Tear VJ, Cao L, et al. Nanoscale dysregulation of collagen structure-function disrupts mechano-homeostasis and mediates pulmonary fibrosis. Elife. 2018;7.pii:e36354.

28. Levental KR, Yu H, Kass L, Lakins JN, Egeblad M, Erler JT, et al. Matrix crosslinking forces tumor progression by enhancing integrin signaling. Cell. 2009;139(5):891-906.

29. Perepelyuk M, Terajima M, Wang AY, Georges PC, Janmey PA, Yamauchi M, et al. Hepatic stellate cells and portal fibroblasts are the major cellular sources of collagens and lysyl oxidases in normal liver and early after injury. Am J Physiol Gastrointest Liver Physiol. 2013;304(6):G605-14.

30. Barker HE, Cox TR, Erler JT. The rationale for targeting the LOX family in cancer. Nat Rev Cancer. 2012;12(8):540-52.

31. Liu SB, Ikenaga N, Peng ZW, Sverdlov DY, Greenstein A, Smith V, et al. Lysyl oxidase activity contributes to collagen stabilization during liver fibrosis progression and limits spontaneous fibrosis reversal in mice. FASEB J. 2016;30(4):1599-609.

32. Iwasaki A, Sakai K, Moriya K, Sasaki T, Keene DR, Akhtar R, et al. Molecular mechanism responsible for fibronectin-controlled alterations in matrix stiffness in advanced chronic liver fibrogenesis. J Biol Chem. 2016;291(1):72-88.

33. Mesarwi OA, Shin MK, Drager LF, Bevans-Fonti S, Jun JC, Putcha N, et al Lysyl oxidase as a serum biomarker of liver fibrosis in patients with severe obesity and obstructive sleep apnea. Sleep. 2015;38(10):1583-91.

34. Grenard P, Bresson-Hadni S, El Alaoui S, Chevallier M, Vuitton DA, Ricard-Blum S. Transglutaminase-mediated cross-linking is involved in the stabilization of extracellular matrix in human liver fibrosis. J Hepatol. 2001;35(3):367-75.

35. Wells RG. Tissue mechanics and fibrosis. Biochim Biophys Acta. 2013;1832(7):884-90. 
36. Orban JM, Wilson LB, Kofroth JA, El-Kurdi MS, Maul TM, Vorp DA Crosslinking of collagen gels by transglutaminase. J Biomed Mater Res A. 2004;68(4):756-62.

37. Fortunati D, Chau DY, Wang Z, Collighan RJ, Griffin M. Cross-linking of collagen I by tissue transglutaminase provides a promising biomaterial for promoting bone healing. Amino Acids. 2014;46(7):1751-61.

38. Mirza A, Liu SL, Frizell E, Zhu J, Maddukuri S, Martinez J, et al. A role for tissue transglutaminase in hepatic injury and fibrogenesis, and its regulation by NF-kappaB. Am J Physiol. 1997;272(2 Pt 1):G281-8.

39. Chen CS, Wu CH, Lai YC, Lee WS, Chen HM, Chen RJ, et al. NF-kappaB-activated tissue transglutaminase is involved in ethanol-induced hepatic injury and the possible role of propolis in preventing fibrogenesis. Toxicology. 2008;246(2-3):148-57.

40. Tatsukawa H, Tani Y, Otsu R, Nakagawa H, Hitomi K. Global identification and analysis of isozyme-specific possible substrates crosslinked by transglutaminases using substrate peptides in mouse liver fibrosis. Sci Rep. 2017;7:45049.

41. Jabłońska-Trypuć A, Matejczyk M, Rosochacki S. Matrix metalloproteinases (MMPs), the main extracellular matrix (ECM) enzymes in collagen degradation, as a target for anticancer drugs. J Enzyme Inhib Med Chem. 2016;31(sup1):177-83.

42. Duarte S, Baber J, Fujii T, Coito AJ. Matrix metalloproteinases in liver injury, repair and fibrosis. Matrix Biol. 2015;44-46:147-56.

43. Knittel T, Mehde M, Grundmann A, Saile B, Scharf JG, Ramadori G. Expression of matrix metalloproteinases and their inhibitors during hepatic tissue repair in the rat. Histochem Cell Biol. 2000;113(6):443-53.

44. Birk DE, Bruckner P. Collagen suprastructures. Top Curr Chem. 2005;247:185-205.

45. Dechêne A, Sowa JP, Gieseler RK, Jochum C, Bechmann LP, El Fouly A, et al. Acute liver failure is associated with elevated liver stiffness and hepatic stellate cell activation. Hepatology. 2010;52(3):1008-16.

46. Latronico T, Mascia C, Pati I, Zuccala P, Mengoni F, Marocco R, et al. Liver Fibrosis in HCV Monoinfected and HIV/HCV Coinfected Patients: Dysregulation of Matrix Metalloproteinases (MMPs) and Their Tissue Inhibitors TIMPs and Effect of HCV Protease Inhibitors. Int J Mol Sci. 2016;17(4):455

47. Qiu H, Zhu Y, Sun Z, Trzeciakowski JP, Gansner M, Depre C, et al. Short communication: vascular smooth muscle cell stiffness as a mechanism for increased aortic stiffness with aging. Circ Res. 2010;107(5):615-9.

48. Jaffar J, Yang SH, Kim SY, Kim HW, Faiz A, Chrzanowski W, et al. Greater cellular stiffness in fibroblasts from patients with idiopathic pulmonary fibrosis. Am J Physiol Lung Cell Mol Physiol. 2018;315(1):L59-65.

49. Sun S, Song Z, Cotler SJ, Cho M. Biomechanics and functionality of hepatocytes in liver cirrhosis. J Biomech. 2014 Jun;47(9):2205-10.

50. Booth AJ, Hadley R, Cornett AM, Dreffs AA, Matthes SA, Tsui JL, et al. Acellular normal and fibrotic human lung matrices as a culture system for in vitro investigation. Am J Respir Crit Care Med. 2012;186(9):866-76.

51. Raizner A, Shillingford N, Mitchell PD, Harney S, Raza R, Serino J, et al. Hepatic inflammation may influence liver stiffness measurements by transient elastography in children and young adults. J Pediatr Gastroenterol Nutr. 2017;64(4):512-7.

52. Seki E, Schwabe RF. Hepatic inflammation and fibrosis: functional links and key pathways. Hepatology. 2015;61(3):1066-79.

53. Cheng T, Liu Q, Zhang R, Zhang Y, Chen J, Yu R, et al. Lysyl oxidase promotes bleomycin-induced lung fibrosis through modulating inflammation. J Mol Cell Biol. 2014;6(6):506-15.

54. Zeng $\mathrm{X}, \mathrm{Xu} \mathrm{C}, \mathrm{He} \mathrm{D}$, Zhang H, Xia J, Shi D, et al. Influence of hepatic inflammation on FibroScan findings in diagnosing fibrosis in patients with chronic hepatitis B. Ultrasound Med Biol. 2015;41(6):1538-44.

55. Perepelyuk M, Terajima M, Wang AY, Georges PC, Janmey PA, Yamauchi M, et al. Hepatic stellate cells and portal fibroblasts are the major cellular sources of collagens and lysyl oxidases in normal liver and early after injury. Am J Physiol Gastrointest Liver Physiol. 2013;304(6):G605-14.

56. Chen G, Lv Y, Guo P, Lin C, Zhang X, Yang L, et al. Matrix mechanics and fluid shear stress control stem cells fate in three dimensional microenvironment. Curr Stem Cell Res Ther. 2013;8(4):313-23.

57. Ma Y, Lin M, Huang G, Li Y, Wang S, Bai G, et al. 3D spatiotemporal mechanical microenvironment: A hydrogel-based platform for guiding stem cell fate. Adv Mater. 2018:e1705911.

58. Pelham RJ Jr, Wang Yl. Cell locomotion and focal adhesions are regulated by substrate flexibility. Proc Natl Acad Sci U S A. 1997;94(25):13661-5.

59. Evans ND, Gentleman E. The role of material structure and mechanical properties in cell-matrix interactions. J Mater Chem B. 2014:2:2345-56.

60. Li Z, Dranoff JA, Chan EP, Uemura M, Sevigny J, Wells RG. Transforming growth factor-beta and substrate stiffness regulate portal fibroblast activation in culture. Hepatology. 2007:46:1246-56.

61. Olsen AL, Bloomer SA, Chan EP, Gaça MD, Georges PC, Sackey B, et al. Hepatic stellate cells require a stiff environment for myofibroblastic differentiation. Am J Physiol Gastrointest Liver Physiol. 2011:301(1):G110-8.

62. Schrader J, Gordon-Walker TT, Aucott RL, van Deemter M, Quaas A, Walsh S, et al. Matrix stiffness modulates proliferation, chemotherapeutic response, and dormancy in hepatocellular carcinoma cells. Hepatology. 2011;53(4):1192-205.

63. Guvendiren M, Perepelyuk M, Wells RG, Burdick JA. Hydrogels with differential and patterned mechanics to study stiffness-mediated myofibroblastic differentiation of hepatic stellate cells. J Mech Behav Biomed Mater. 2014;38:198-208.

64. Caliari SR, Perepelyuk M, Cosgrove BD, Tsai SJ, Lee GY, Mauck RL, et al. Stiffening hydrogels for investigating the dynamics of hepatic stellate cell mechanotransduction during myofibroblast activation. Sci Rep. 2016;6:21387.

65. Caliari SR, Perepelyuk M, Soulas EM, Lee GY, Wells RG, Burdick JA. Gradually softening hydrogels for modeling hepatic stellate cell behavior during fibrosis regression. Integr Biol (Camb). 2016;8(6):720-8

66. Saums MK, Wang W, Han B, Madhavan L, Han L, Lee D, et al. Mechanically and chemically tunable cell culture system for studying the myofibroblast phenotype. Langmuir. 2014;30:5481-7.

67. Bomo J, Ezan F, Tiaho F, Bellamri M, Langouët S, Theret N, et al. Increasing 3D matrix rigidity strengthens proliferation and spheroid development of human liver cells in a constant growth factor environment. J Cell Biochem. 2016;117(3):708-20.

68. Ma X, Yu C, Wang P, Xu W, Wan X, Lai CSE, et al. Rapid 3D bioprinting of decellularized extracellular matrix with regionally varied mechanical properties and biomimetic microarchitecture. Biomaterials. 2018;185:310-21.

69. Liu L, You $Z$, Yu H, Zhou L, Zhao $H$, Yan $X$, et al. Mechanotransduction-modulated fibrotic microniches reveal the contribution of angiogenesis in liver fibrosis. Nat Mater. 2017;16(12):1252-61.

70. Gurtner GC, Padmanabhan J. Disease models: Method in the madness of fibrosis. Nat Mater. 2017;16(12):1176-7.

71. Wipff PJ, Rifkin DB, Meister JJ, Hinz B. Myofibroblast contraction activates latent TGF-beta1 from the extracellular matrix. J Cell Biol. 2007;179(6):1311-23.

72. Hinz B. Tissue stiffness, latent TGF-beta1 activation, and mechanical signal transduction: implications for the pathogenesis and treatment of fibrosis. Curr Rheumatol Rep. 2009;11(2):120-6.

73. Zhou Y, Hagood JS, Lu B, Merryman WD, Murphy-Ullrich JE. Thy-1-integrin alphav beta5 interactions inhibit lung fibroblast contraction-induced latent transforming growth factor-beta1 activation and myofibroblast differentiation. J Biol Chem. 2010;285(29):22382-93.

74. Huang X, Yang N, Fiore VF, Barker TH, Sun Y, Morris SW, et al. Matrix stiffness-induced myofibroblast differentiation is mediated by intrinsic mechanotransduction. Am J Respir Cell Mol Biol. 2012;47(3):340-8.

75. Marinković A, Liu F, Tschumperlin DJ. Matrices of physiologic stiffness potently inactivate idiopathic pulmonary fibrosis fibroblasts. Am J Respir Cell Mol Biol. 2013;48(4):422-30.

76. Tomasek JJ, Vaughan MB, Kropp BP, Gabbiani G, Martin MD, Haaksma CJ, et al. Contraction of myofibroblasts in granulation tissue is dependent on Rho/rho kinase/myosin light chain phosphatase activity. Wound Repair Regen. 2006;14(3):313-20.

77. Dou C, Liu Z, Tu K, Zhang H, Chen C, Yaqoob U, et al. P300 Acetyltransferase mediates stiffness-induced activation of hepatic stellate cells into tumor-promoting 2018;154(8):2209-2221.e14.

78. Panciera T, Azzolin L, Cordenonsi M, Piccolo S. Mechanobiology of YAP and TAZ in physiology and disease. Nat Rev Mol Cell Biol. 2017;18(12):758-70.

79. Brusatin G, Panciera T, Gandin A, Citron A, Piccolo S. Biomaterials and engineered microenvironments to control YAP/TAZ-dependent cell behaviour. Nat Mater. 2018;17(12):1063-75.

80. Mannaerts I, Leite SB, Verhulst S, Claerhout S, Eysackers N, Thoen LF, et al. The Hippo pathway effector YAP controls mouse hepatic stellate cell activation. J Hepatol. 2015;63(3):679-88.

81. Santos A, Lagares D. Matrix stiffness: the conductor of organ fibrosis. Curr Rheumatol Rep. 2018;20(1):2.

82. Liu F, Lagares D, Choi KM, Stopfer L, Marinković A, Vrbanac V, et al. Mechanosignaling through YAP and TAZ drives fibroblast activation and fibrosis. Am J Physiol Lung Cell Mol Physiol. 2015; 308(4): L344-57.

83. Rosin NL, Sopel MJ, Falkenham A, Lee TD, Légaré JF. Disruption of collagen homeostasis can reverse established age-related myocardial fibrosis. Am J Pathol. 2015;185(3):631-42.

84. Barry-Hamilton V, Spangler R, Marshall D, McCauley S, Rodriguez HM, Oyasu M, et al. Allosteric inhibition of lysyl oxidase-like-2 impedes the development of a pathologic microenvironment. Nat Med. 2010;16(9):1009-17.

85. Ikenaga N, Peng ZW, Vaid KA, Liu SB, Yoshida S, Sverdlov DY, et al. Selective targeting of lysyl oxidase-like 2 (LOXL2) suppresses hepatic fibrosis progression and accelerates its reversal. Gut. 2017;66(9):1697-708.

86. Liu X, Zhao $Y$, Gao J, Pawlyk B, Starcher B, Spencer JA, et al. Elastic fiber homeostasis requires lysyl oxidase-like 1 protein. Nat Genet. 2004;36(2):178-82.

87. Zhao W, Yang A, Chen W, Wang P, Liu T, Cong M, et al. Inhibition of lysyl oxidase-like 1 (LOXL1) expression arrests liver fibrosis progression in cirrhosis by reducing elastin crosslinking. Biochim Biophys Acta Mol Basis Dis. 2018;1864:1129-37.

88. Huang S, Wettlaufer SH, Hogaboam C, Aronoff DM, Peters-Golden M. Prostaglandin E(2) inhibits collagen expression and proliferation in patient-derived normal lung fibroblasts via E prostanoid 2 receptor and cAMP signaling. Am J Physiol Lung Cell Mol Physiol. 2007;292(2):L405-13.

89. Okazaki I, Maruyama K. Collagenase activity in experimental hepatic fibrosis. Nature. 1974; 252:49-50.

90. Discher DE, Janmey P, Wang YL. Tissue cells feel and respond to the stiffness of their substrate. Science. 2005;310(5751):1139-43.

91. Herrera J, Henke CA, Bitterman PB. Extracellular matrix as a driver of progressive fibrosis. J Clin Invest. 2018;128(1):45-53. 
92. Lampi MC, Reinhart-King CA. Targeting extracellular matrix stiffness to attenuate disease: From molecular mechanisms to clinical trials. Sci Transl Med. 2018;10(422): eaao0475.

93. Htwe SS, Cha BH, Yue K, Khademhosseini A, Knox AJ, Ghaemmaghami AM. Role of Rho-associated coiled-coil forming kinase isoforms in regulation of stiffness-induced myofibroblast differentiation in lung fibrosis. Am J Respir Cell Mol Biol. 2017;56(6):772-83.

94. Zhou Y, Huang X, Hecker L, Kurundkar D, Kurundkar A, Liu H, et al. Inhibition of mechanosensitive signaling in myofibroblasts ameliorates experimental pulmonary fibrosis. J Clin Invest. 2013;123(3):1096-108.

95. Zhang K, Chang Y, Shi Z, Han X, Han Y, Yao Q, et al. $\omega-3$ PUFAs ameliorate liver fibrosis and inhibit hepatic stellate cells proliferation and activation by promoting YAP/TAZ degradation. Sci Rep. 2016;6:30029.

96. Henderson NC, Mackinnon AC, Farnworth SL, Poirier F, Russo FP, Iredale JP, et al. Galectin-3 regulates myofibroblast activation and hepatic fibrosis. Proc Natl Acad Sci U S A. 2006;103(13):5060-5.

97. Torr EE, Ngam CR, Bernau K, Tomasini-Johansson B, Acton B, Sandbo N. Myofibroblasts exhibit enhanced fibronectin assembly that is intrinsic to their contractile phenotype. J Biol Chem. 2015;290(11):6951-61.

98. Lin YC, Sung YK, Jiang X, Peters-Golden M, Nicolls MR. Simultaneously targeting myofibroblast contractility and extracellular matrix cross-linking as a therapeutic concept in airway fibrosis. Am J Transplant. 2017;17(5):1229-41. 